\title{
Where to go: Computational and visual what-if analyses in soccer
}

\author{
Manuel Stein ${ }^{a}$, Daniel Seebacher ${ }^{a}$, Rui Marcelino (D) ${ }^{b, c}$, Tobias Schreck ${ }^{d}$, Michael Grossniklaus (D) , Daniel A. Keim ${ }^{a}$ \\ and Halldor Janetzko
}

\begin{abstract}
aDepartment of Computer and Information Science, University of Konstanz, Konstanz, Germany; bepartment of Sports Sciences, Research Center in Sports Sciences, Health Sciences and Human Development, CIDESD, CreativeLab Research Community, Vila Real, Portugal; 'University Institute of Maia, Maia, Portugal; 'Institute of Computer Graphics and Knowledge Visualization, Graz University of Technology, Graz, Austria; ${ }^{\text {eDepartment of }}$ Computer Science, Lucerne University of Applied Sciences and Arts, Lucerne, Switzerland
\end{abstract}

\begin{abstract}
To prepare their teams for upcoming matches, analysts in professional soccer watch and manually annotate up to three matches a day. When annotating matches, domain experts try to identify and improve suboptimal movements based on intuition and professional experience. The high amount of matches needing to be analysed manually result in a tedious and time-consuming process, and results may be subjective. We propose an automatic approach for the realisation of effective region-based what-if analyses in soccer. Our system covers the automatic detection of region-based faulty movement behaviour, as well as the automatic suggestion of possible improved alternative movements. As we show, our approach effectively supports analysts and coaches investigating matches by speeding up previously time-consuming work. We enable domain experts to include their domain knowledge in the analysis process by allowing to interactively adjust suggested improved movement, as well as its implications on region control. We demonstrate the usefulness of our proposed approach via an expert study with three invited domain experts, one being head coach from the first Austrian soccer league. As our results show that experts most often agree with the suggested player movement (83\%), our proposed approach enhances the analytical capabilities in soccer and supports a more efficient analysis.
\end{abstract}

\section{KEYWORDS}

Visual analytics; sports analytics; soccer analytics; information visualisation

\section{Introduction}

European football (soccer) has a major social impact - more than 3 billion spectators were expected to watch the FIFA World Cup 2018 in Russia (FIFA, 2018) - as well as an economic impact (Dobson \& Goddard, 2001). This most recent World Cup was set to be the most technologically advanced to date (BBC, 2018), and the amount of data generated in each game has seen an exponential growth compared to previous events. Traditionally, data collected from soccer events comprised audio, video, and manually curated statistical data. Nowadays, due to various recent improvements in sensor technologies, with the possibility to collected data with radio frequency systems (Frencken, Lemmink, \& Delleman, 2010; Sathyan, Shuttleworth, Hedley, \& Davids, 2012), differential and non-differential GPS units (Johnston, Watsford, Kelly, Pine, \& Spurrs, 2014; Varley, Fairweather, \& Aughey, 2012; Waldron, Worsfold, Twist, \& Lamb, 2011), or semi-automated computer vision systems (Redwood-Brown, Cranton, \& Sunderland, 2012; Stein et al., 2018; Valter, Adam, Barry, \& Marco, 2006), many more kinds of data can be captured, including the positional data ( $x$ - and $y$-coordinates) of the players and of the ball. Research and practical application resulting from positional data are traditionally focused on the description of the individual physical demands and impacts on players, both at training and in competition settings (Abade, Gonçalves, Leite, \& Sampaio, 2014; Castellano,
Puente, Echeazarra, Usabiaga, \& Casamichana, 2016; Krustrup, Mohr, Ellingsgaard, \& Bangsbo, 2005). However, there is a growing interest in the study of the collective team behaviours, using the spatiotemporal characteristics of players, with the propose of assess the features of team tactics or styles of play (Alexander, Spencer, Mara, \& Robertson, 2019; Clemente, Couceiro, Martins, Mendes, \& Figueiredo, 2013; Folgado, Bravo, Pereira, \& Sampaio, 2019; Gonçalves, Marcelino, Torres-Ronda, Torrents, \& Sampaio, 2016). Recently, an exploratory prototype to perform datadriven visual performance analysis in soccer was proposed by Benito Santos and colleagues (Benito Santos, Theron, Losada, Sampaio, \& Lago-Peñas, 2018), in which some group collective behaviour analysis were explored. The access to this new set of data and the possibility of processing the data in an integrated way has allowed the emergence of new analysis models of sport performance (Link, 2018; Rein \& Memmert, 2016; Stein et al., 2017). In competitive team sports, improving performance is crucial, and clubs regularly perform interactive video analysis. The goal of this video-analysis is to identify strengths and weaknesses of both, the own and the opposing team, with the ultimate goal to gain advantages in the competition.

Previous studies, for example, determined appropriate actions, e.g., passes to perform, depending on the position of the remaining players of the own and opposing teams (Rein, Raabe, \& Memmert, 2017). Lucey and co-authors 


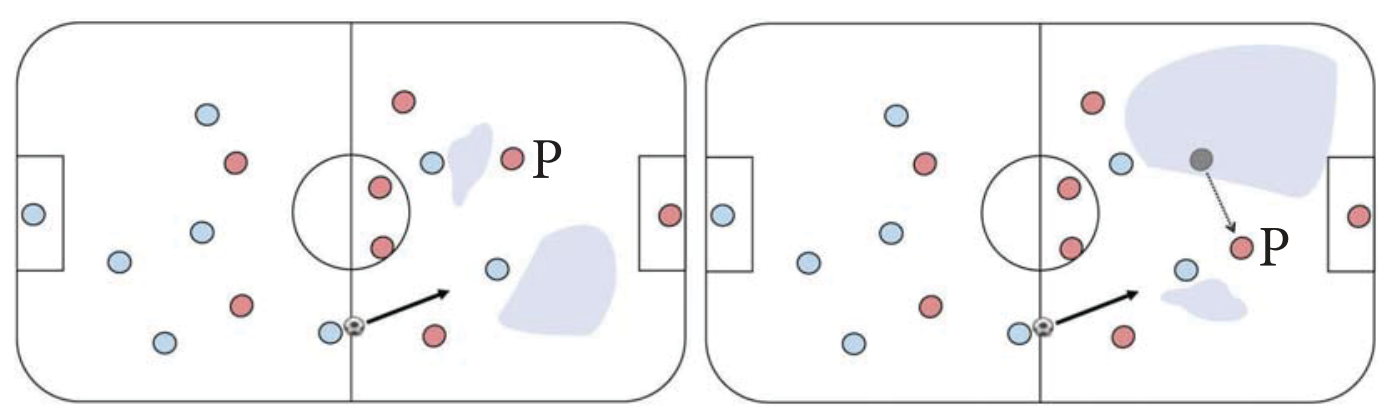

(a) Observed Movement

(b) Wrongly suggested alteration

Figure 1. The automatic assessment of how a player should have moved to receive a better outcome of a situation is difficult. An alteration of real movement can even result in a worse situation, as e.g., shrinking the lower free space (a) by simply moving the red player $\mathbf{P}$ results in enlarging the upper free space (b). Please note that we reduced the number of players in this figure for legibility reasons.

conducted several data-driven studies, analysing how a team is likely to defend, through showing the "ghost" players trajectory analysis in basketball (Seidl, Cherukumudi, Hartnett, Carr, \& Lucey, 2018; Sha et al., 2018) and football (Le, Carr, Yue, \& Lucey, 2017). In one of their studies the authors ask: "What if a coach did not have to rely solely on intuition, but instead could see instantly how the defending team is likely to respond?" (Seidl et al., 2018, p. 1). In video-based analysis of soccer matches, findings are presented to the team members, coaches, managers, and other stakeholders in form of manually annotated video clips. During team briefings, coaches and analysts pause these video recordings based on their experiences at certain situations to explain suboptimal movement behaviour as well as to indicate where players could have moved to improve the situation. In this paper, we introduce a novel interactive visual analysis method supporting positional what-if analysis in soccer. To make this possible, our presented approach supports the experts in several parts of the analysis process. Initially, suggestions are made for situations of interest, which should be investigated in more detail. In these situations, our algorithm can then perform the what-if analysis and optimise the players' positions. Additionally, we provide visualisations for assessment that help users to understand the expected effects of the repositioning of the players. Finally, we show that our technique enables domain experts to create realistic and expressive analyses of alternative movement options.

The effectiveness of what-if analyses strongly depends on the solutions to two major challenges: (1) the identification of a point in time at which the movement outcome can be positively influenced and (2) the prediction ability for alternative movement behaviour. Identifying a point in time where we can positively influence movement behaviour is inherently difficult due to two aspects: the identified point of time to optimise the movement behaviour is either too close to the begin of the observed period of time (i.e., there is not enough time left to optimise the movement) or the begin point of time is identified too far in the past and the predicted movement is not reliable anymore (i.e., all players have too much time to react resulting in a high variability). In between both temporal extremes is an intermediate sweet spot allowing enough time to optimise the movement outcome, and still do stable outcome prediction. This sweet spot is unfortunately not always the same, and a fully automatic identification is not possible. In Section 2.2, we propose an approach to detect the most promising point in time as a starting point for an alternative movement prediction. This serves to improve the detected arguable movement behaviour, additionally allowing the analyst to explore the temporal neighbourhood as well. The prediction quality relies on the context inclusion in the movement prediction algorithm. Currently, there exist no way to validate the hypotheses of what-if scenarios based on real player movement data causing tedious and error-prone manual analyses. Experts have to rely on their own experience while looking for suboptimal movement behaviour as well as alternative movement possibilities.

Computational assessment of alternative movement and potential outcome of a situation are challenging problems. An alternative location of players can easily result in a worse situation as depicted in Figure 1. Consequently, both domain experts and algorithms have to take the situation context into account, assessing and changing the positions of players. Therefore, a proper algorithmic solution needs to make sure that proposed local alterations do not result in a worse situation. Thus, we incorporate the collective movement of both teams into the assessment and the proposal of alternatives, e.g., by evaluating the creation and destruction of free spaces. Processing large amounts of player movement data, our system proposes how players could have altered their movement in respective situations based on real underlying data.

Based on the above considerations, and on requirements obtained by interviews with several coaches and analysts from international first league soccer clubs, we state the following research hypotheses:

(1) The combination of automatic and interactive visual analysis is beneficial for the detection when and where suboptimal or faulty movement behavior occurs, incorporating all relevant data;

(2) Faulty movement detection and generation of what-if scenarios can be algorithmically modeled and computed efficiently;

(3) The synergetic automatic and visual-interactive approach effectively supports and improves the work of coaches and video analysts. 
We contribute to the state-of-the-art by enabling analysts to validate hypotheses automatically as well as interactively by manually repositioning players via drag-and-drop on a visual pitch. The impact of the change is re-analysed and visualised, e.g., in form of free spaces and helps analysts to identify improvement options to use during coaching. This combined automatic and interactive approach enables new forms of interactive video analysis, and is especially suited also for discussion and presentation within a team. We also evaluate our technique with respect to the research hypotheses through several expert studies, including feedback from coaches and analysts of professional soccer clubs.

\section{Methods}

\subsection{Data}

The data we use for our research on region-based what-if analysis are provided by STATS (https://www.stats.com/) and consist of overall 65 matches from different first leagues and competitions. The data is available in XML format and contains meta-information of the match such as the competition, the date, as well as detailed information about the teams involved and their players. The detailed information being crucial for our research are event data and position data of players and ball. The position data of the individual players and the ball are available in a time resolution of 100 milliseconds and provide information about their $x$ - and $y$-positions on the soccer pitch. Additionally available event data are usually associated with the soccer ball, for example, when a shot, an offside, or a foul occurred. These events can be used to filter for interesting situations such as a shot at the goal, but also to segment and structure the game, e.g., an attack situation consisting of a dribbling, a pass into free space and a subsequent shot.

\subsection{Procedures}

We consider match situations - including significant or match deciding events involving faults - that lead to a major disadvantage of one team as basis to perform what-if analyses. For these situations, analysts are usually highly interested in (a) finding faulty movement behaviour (Section 2.2.1) as well as (b) providing alternatives to the team in order to avoid these faults (Section 2.2.2). While (b) reflects the what-if analyses, (a) is a necessary input to perform such analyses. Consequently, we consider the detection of faulty movement behaviour as an essential first step for a successful what-if analysis. We focus in this work on defensive behaviour only, as a good defence is crucial for a successful team. Ball possession is highly depending on a productive defending behaviour and faulty positioning in defending phases can be fatal.

To support analysts in investigating matches, our designed technique has to be transparent, traceable, as well as adjustable to the needs of the analyst. Therefore, we rely on criteria describing soccer movement and high-level features such as interaction spaces and free spaces. Furthermore, we present a transparent rule-based approach that produces explainable and interpretable results. This is an intentional design decision to avoid often used black-box techniques such as Deep Learning methods. While widely used black-box machine learning techniques can achieve remarkable results, they do so at the expense of explainability and interpretability. There are some first efforts to make such models comprehensible, but some important scientific questions remain unanswered, which can lead to practical, ethical and trust issues. A detailed description and discussion of this problem can be found in the survey by (Guidotti et al., 2019).

As described in Section 2.1, we do not have any information of the third dimension and would assume players and ball being on one plane. However, our methods are not depending on any information of the z-coordinate as they just describe which player can reach a location first. Algorithms for the region-based what-if analyses decreasing free spaces, increasing pressing, or relocating players are also not dependent on more than the $\mathrm{x}$ - and $\mathrm{y}$-coordinates.

\subsubsection{Detection of faulty movement behaviour}

There exist a large variety of possible faulty movement behaviours ranging from lost header duels, to players positioning themselves wrongly on the pitch. However, as our data (see Section 2.1) consists mainly of two-dimensional $x$ - and $y$-coordinates, we restrict ourselves on the detection of movementand region-based faulty behaviour. Free space analysis as, for example, proposed by Stein et al. (2016) has proven to be a powerful concept to estimate the regions that certain players or whole teams are potentially controlling. An overview of our resulting approach for the detection of faulty movement behaviour can be seen in Figure 2. Our approach can either be applied to the whole match, or to previously chosen situations, e.g., by enabling analysts to define

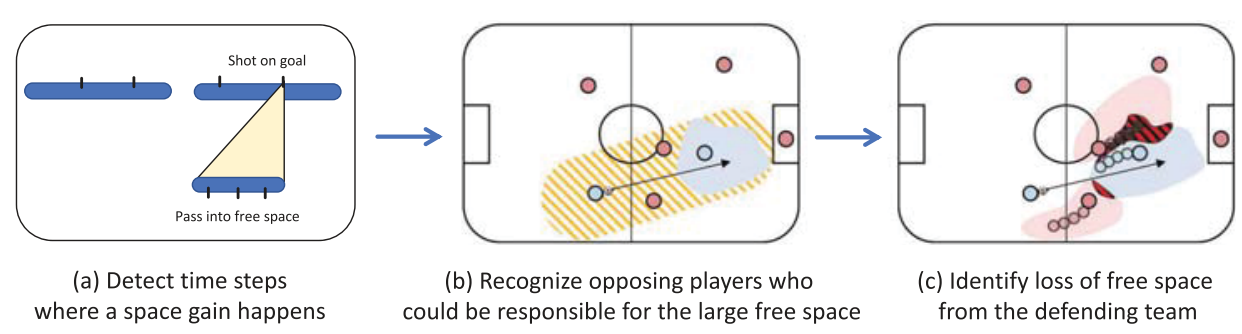

Figure 2. Detecting faulty movement behaviour is non-trivial. For our approach, we first identify time steps where a large free space is created, e.g., during a pass into a large free space. Afterwards, we identify opposing players who were close to the ball trajectory as possible responsible candidates for the control of this free space. These candidates are further analysed by extracting the area lost to the attacking player. The larger the loss of free space, the more likely it is that this opposing player has moved incorrectly. 
important events that have to be included such as shot on goal events. For each given interesting situation, we afterwards need to verify whether this situation could have been caused by faulty movement behaviour. Consequently, we iterate backwards starting from the end of the situation, inspecting the collective movement of all individual players at each point in time. We calculate all free spaces for each time step in a predefined time period prior to the interesting situation (Figure 2(a)), specifically looking at passes in large open free spaces. Consequently, we assess which opposing players lose the most free space to the player of the attacking team who receives the pass in the created free space (Figure $2(b, c)$ ). These defending players are our candidates for potential faulty movement behaviour and also serve as the starting point for the what-if analysis. In the following, we describe the detection of faulty movement behaviour in detail. All processing steps can be configured using several parameters and can be adjusted by the user if needed.

1. Detection of free spaces. (Figure 2(a)) We use the definition of Stein et al. (2016), where free spaces are defined as the regions that a player can reach before any other player, considering various attributes such as distance, current speed, and heading. In our case, we calculate free spaces for each player for each time step in a predefined time period before the occurrence of given interesting situations, in our case shot on goal events. The duration of the timespan can be set by the analyst, for example, the 30 seconds before a shot on goal event. Within this selected time period, we subsequently identify all passes into large free spaces as these passes are used to overcome opposing players.

2. Detection of involved players. (Figure 2(b)) After the detection of free spaces, we identify the involved players which may have shown false movement behaviour. To detect these players, we cheque which players are within a certain distance to the ball positions during the pass as displayed in Figure 2(b). Realistically, only players near the ball would have been able to intercept the ball or reduce the free space of the attacking players. The hatched area in Figure 2(b) represents the distance around the ball which is used to identify involved defending players. After discussion with experts, as well as after reviewing the first preliminary results, we experimentally set this value to $8.75 \mathrm{~m}$. Alterations of this value affect which players are considered for the identification of faulty movement behaviour. Too high values include too many players, which may result in players being analysed who did not participate in the respective game situation. On the other hand, if the value is set too low, important involved players may not be considered.

3. Identification of faulty movement behaviour. (Figure 2(c)) In the last step, we examine which players may have performed faulty movement behaviour in the given match situation. Consequently, we take the players of the defending team that we identified in the previous step and calculate, for any time step between two seconds before a pass and its reception, how much free space each of them lost to the ball receiving player of the attacking team. The identified difference corresponds to the region the attacker gained control over by her or his movement behaviour. Conversely, we recognise when a defending player potentially shows a faulty movement behaviour based on a large loss of space. Additionally, as free spaces depend on all moving players on the field and can thus change quickly, we calculate the duration the defending player loses free space to the attacking player. Afterwards, the identified candidates for faulty movement behaviour are assessed based on duration, area of free space lost, as well as distance to the time step where the pass was received by the attacking player.

\subsubsection{Region-based what-if analyses}

After identifying situations containing potentially faulty movement behaviour, we focus on enabling an effective what-if analysis for these cases. It is important for coaches and analysts to recognise and present faulty movement behaviour and movement alternatives to the team to improve team performance. Using our proposed what-if analysis, we show how a physically realistic position change of a player could have affected the current situation. Here, our goal is to maximise important regions which are under the control of a team, for example, near the own defenders by shifting the player who makes the mistake to a realistic and improved position. Exploring what-if scenarios, however, is very complex, as each change might have unforeseen implications. Simply moving players closer to the ball or into opponents' free spaces could result in an overall loss of the free space of their team, as shown in Figure 1, which is potentially worse. Furthermore, a player should theoretically be able to reach a proposed position respecting physics. We consider this as an optimisation problem assuming that players are constantly trying to reach their optimum location concerning all other player positions and respective goals.

We distinguish between a user-driven, interactive approach as well as an automatic approach, presenting previously unknown alternatives to perform what-if analyses. For the first approach, we allow the user to interactively inspect the implications of possible changes or alternative positions for the current situation by a dragand-drop interaction with any player. Once a player has been selected by the user (mouse clicking), our system recalculates free spaces during every position change (mouse moving) and calculates optimised, realistic player trajectories for defending players on their path to the identified optimal position (Figure 3),

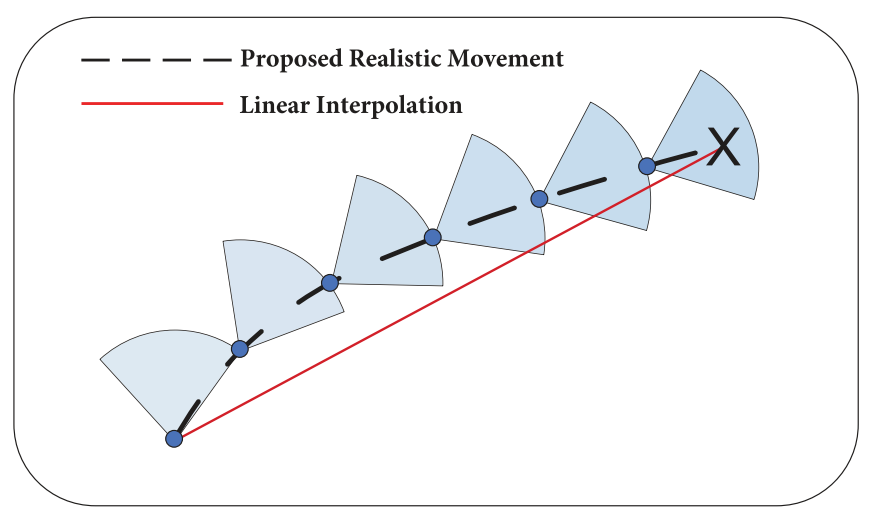

Figure 3. To enable domain experts to properly assess the proposed optimal position, we provide a realistic player trajectory. It is representing how the player could have moved to the identified optimal position based on the player's interaction space (dashed line), based on an appropriate movement and interaction (Stein et al., 2016). 
thus, enabling experts to compare, confirm, or reject their hypotheses with respect to the displayed results. For our automatic approach, we calculate an improved position for a defending player with faulty movement behaviour as displayed in Figure 4. Afterwards, our system again proposes optimised, realistic player trajectories for defending players. Eventually, a complete overview of the workflow of our implemented what-if analysis can be seen in Figure 5. Below, we describe every step of our automatic approach in detail.

1. Calculate improved player movement. The starting point of our automatic what-if analysis is the first time step of the identified faulty movement behaviour, calculated as previously described in Section 2.2.1. We now define which region a defending player could have reached between faulty movement behaviour and the pass into the arising free space by placing an invisible, dynamic grid with the defending player at the centre (Figure 4 left) on the soccer pitch. The side length of the individual cells is experimentally set to 0.5 metres, as this is approximately the space that one person occupies. The side length can of course be set differently, however, there is a trade-off between computational complexity and accuracy. For each cell, we calculate the free spaces if the defending player would have been at this position covering different speeds and running directions and, therefore, update the controlled regions of all players. We arses the quality of a position change by calculating how much the free space of the attacking player towards the goal is being reduced as depicted in the middle of Figure 4. After assessing each cell, the defending player is moved towards the identified optimal position corresponding to the position with the best rating (Figure 4 right).
To enable domain experts to properly assess the proposed optimal position, we also focus on providing a realistic player trajectory representing how the player could have moved to the identified optimal position. As players are bound by the laws of physics and, consequently, speed and direction influence their running behaviour (see Interaction Spaces of Stein et al. (2016)), we provide an interpolation based on the possible movement of a player instead of, for example a simple linear interpolation between past and optimal player position. Figure 3 demonstrates how we calculate the movement to a determined optimal player position. The red line in Figure 3 shows a linear interpolation between player and calculated optimal position, proposing unrealistic movement as humans in the real world, bound to the laws of physics, cannot change their running direction immediately. To obtain a more realistic player trajectory, we introduce a movement calculation based on a player's interaction space as described in Stein et al. (2016). As soon as the identified defending player starts to lose room control towards the attacking opposing player (so right at the beginning of the faulty movement behaviour), we visualise how the defending player moves to the optimal position by calculating the player's interaction space, searching for the nearest point between interaction space and optimal position. Next, we determine the new running direction of the player by calculating the angle between the current and the detected positions. These steps are repeated until the optimal position is reached. The detected player positions are represented as blue dots in Figure 3.

2. Calculate further movement behaviour until the pass is played. So far, we proposed a realistic player trajectory aiming at the calculated optimal position which mostly reduces the

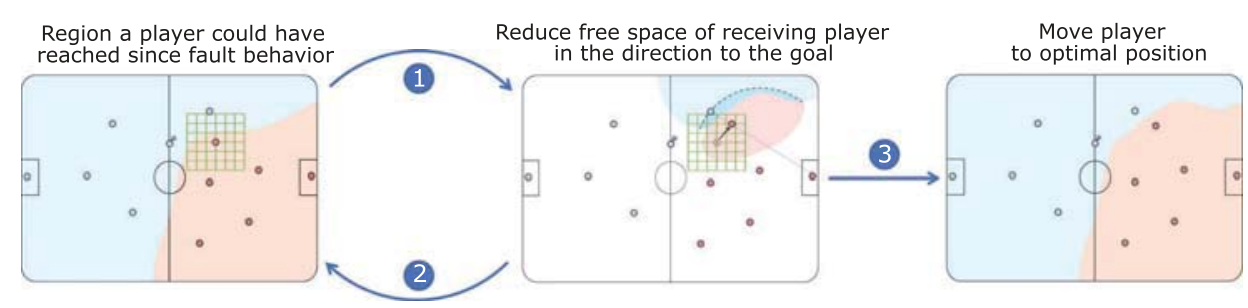

Figure 4. After we detected which defending players were causing a dangerous opposing free space by losing important room control, we calculate optimised player positions. We calculate the region each player could have reached since the initially faulty movement behaviour (left). Afterwards, we arses each possible position determining the optimal player position based on how much the free space of the attacking opposing player can be reduced towards the own goal (middle). Eventually, each detected player is shifted towards the region with the best result (right). In this schematic picture, only six players of each team are depicted for visual clarity. Our algorithm considers all players from both teams.

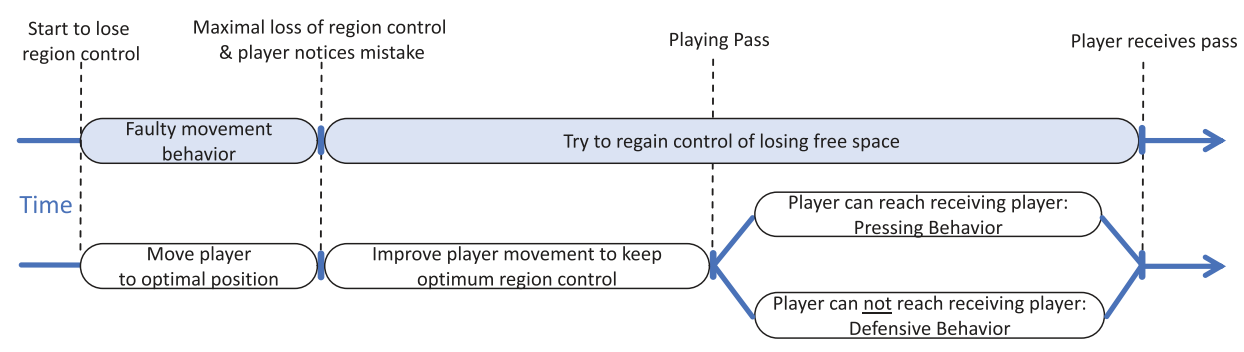

Figure 5. Every step of our workflow for the implemented what-if analysis. Our what-if analysis starts at the moment an identified defending player starts to lose room control. Afterwards, we constantly optimise the defending player's movement trajectory to reduce the free space of the ball receiving attacking player towards the own goal. Eventually, during the pass to the attacking player, our system either proposes pressing behaviour or a more defensive behaviour, based on whether the attacking player can be reached in time. 
free space and, therefore, the room control of the attacking player at the point in time when the originally detected faulty movement behaviour occurred. Still, the situation might not have finished yet as the attacking team possibly did not yet try to pass in the, initially large, free space of the attacking player. To demonstrate how the defending player could have stayed in control of the important space between attacking player and own goal until the pass is played, we again move the player to the optimal position at the time of the pass, calculated as previously described.

\section{Which behaviour should the moved player show during} the pass? The last step is to determine the movement behaviour of the defending player, right at the moment of the pass towards the attacking player. The defending player can either stay defensive, or try to attack the opposing player. Increasing the pressure on the attacking, ball receiving player can result in a higher probability of gaining possession of the ball. A more defensive behaviour allows to keep room control towards the own goal and might be useful, e.g., when a technically high-skilled attacking player is currently in ball possession. To determine whether the system should suggest pressing or a more defensive play style, we calculate whether the defending player could reach the attacking player, at least at the moment of ball reception. If this is the case, our system proposes the defending player to put pressure on the attacking ball receiving player. Otherwise, our system proposes a more defensive behaviour.

3a. Pressing Behaviour. A defending player is putting pressure on an attacking opposing player if the defending player is moving towards the attacking player to disturb or stop the ball reception. In our system, we let the defending player run towards the attacking player who is about to receive the ball. We reuse the previously introduced calculation from Figure 3 to compute a realistic movement. Additionally, we pay special attention to avoid players running directly onto the other player, but instead moving slightly in front of the attacking player, constantly blocking the angle and path to the own goal.

3b. Defensive Behaviour. If the defending player is expected to show a more defensive behaviour, our system keeps the defending player moving on an optimal path which is recalculated and updated every time step. Consequently, the attacking player's free space and movement is further being reduced. We realise this by reusing our previously introduced approach from Figure 4 with the difference that we constantly use the spatial coordinates of the attacking player who is about to receive the ball instead of the ball.

We evaluated our proposed approach with three invited domain experts. The first expert (Expert One in Table 1) is a former professional, international first league soccer player and is currently working as head coach of an Austrian first league (Austrian Football Bundesliga) team. In addition to being a certified first league coach, he is also a certified match analyst. The second expert (Expert Two in Table 1) is playing soccer actively for more than 26 years and has been working as a coach for 13 years. Until recently, he has been working for the German soccer club FC Bayern München in the youth department. The third expert (Expert Three in Table 1) has been an active soccer player for 16 years and is working as coach in the youth sector. Moreover, he is currently studying sport sciences analysing the annotation of tactical movement
Table 1. We invited three domain experts in order to test our proposed what-if analysis with real match data from a European international soccer club competition. Our system identified 14 situations, where an assumed faulty movement behaviour lead to a successful shot on goal event. All experts agreed with the 14 situations being classified as faulty movement behaviour. As further shown in the table, the invited experts mainly agreed with our following suggested improved player movement.

\begin{tabular}{lccc}
\hline Scene & Expert One & Expert Two & Expert Three \\
\hline 1 (Shot 1, 2 Passes Before Shot) & $\checkmark$ & $\checkmark$ & $\checkmark$ \\
2 (Shot 1, 3 Passes Before Shot) & $\checkmark$ & $\checkmark$ & $\boldsymbol{X}$ \\
3 (Shot 5, 1 Pass Before Shot) & $\checkmark$ & $\checkmark$ & $\checkmark$ \\
4 (Shot 6, 1 Pass Before Shot) & $\checkmark$ & $\checkmark$ & $\checkmark$ \\
5 (Shot 8, 1 Pass Before Shot) & $\checkmark$ & $\boldsymbol{x}$ & $\boldsymbol{X}$ \\
6 (Shot 9, 5 Passes Before Shot) & $\checkmark$ & $\checkmark$ & $\checkmark$ \\
7 (Shot 9, 6 Passes Before Shot) & $\checkmark$ & $\checkmark$ & $\checkmark$ \\
8 (Shot 9, 8 Passes Before Shot) & $\checkmark$ & $\checkmark$ & $\boldsymbol{X}$ \\
9 (Shot 11, 6 Passes Before Shot) & $\boldsymbol{x}$ & $\checkmark$ & $\checkmark$ \\
10 (Shot 11, 7 Passes Before Shot) & $\checkmark$ & $\checkmark$ & $\checkmark$ \\
11 (Shot 13, 2 Passes Before Shot) & $\boldsymbol{x}$ & $\checkmark$ & $\boldsymbol{X}$ \\
12 (Shot 20, 3 Passes Before Shot) & $\checkmark$ & $\checkmark$ & $\checkmark$ \\
13 (Shot 22, 1 Pass Before Shot) & $\checkmark$ & $\checkmark$ & $\checkmark$ \\
14 (Shot 22, 3 Passes Before Shot) & $\checkmark$ & $\checkmark$ & $\checkmark$ \\
Accuracy & $\mathbf{8 5 . 7 1 \%}$ & $\mathbf{9 2 . 8 5 \%}$ & $\mathbf{7 1 . 4 2 \%}$ \\
\hline
\end{tabular}

behaviour in soccer matches. To prepare their teams for upcoming matches, all of the invited experts watch and annotate up to three matches a day using state-of-the-art video tagging systems such as Sportscode or Dartfish. Briefly, these video tagging systems enable analysts to interactively define tags (also known as events) being set when a specific key is pressed. These systems do not allow to work with the underlying movement data, so their important search for regionbased faulty movement behaviour is performed completely manually by watching videos.

For our user study, we separately invited all experts to use our system for the detection of faulty movement behaviour as well as our proposed resulting region-based what-if analyses. All capabilities of our proposed system were explained to each expert. Afterwards, we loaded our proposed approach using a match from a European international soccer club competition. For the given match, our system proposed 14 situations, where an assumed faulty movement behaviour lead to a successful shot on goal event. For each assumed faulty movement behaviour, we asked each expert separately to verify whether the classification as faulty movement behaviour was correct as well as whether our automatically what-if analysis proposed an improved (ideal) solution. Every expert was allowed to spend as much time as desired with each identified faulty movement behaviour. During each user study, we encouraged the experts to express ad-hoc comments via the Thinking-Aloud (Boren \& Ramey, 2000; Ericsson \& Simon, 1984) method. Afterwards, we performed an interview with each expert in order to gather feedback about the usefulness, completeness as well as applicability of our system. In addition, we asked for suggestions for improvements.

\section{Results}

The results of both expert studies are very promising. The experts identified faulty movement behaviour in every proposed situation. An exemplary situation the experts approved can be seen in Figure 6. Furthermore, all three domain experts agreed with our 


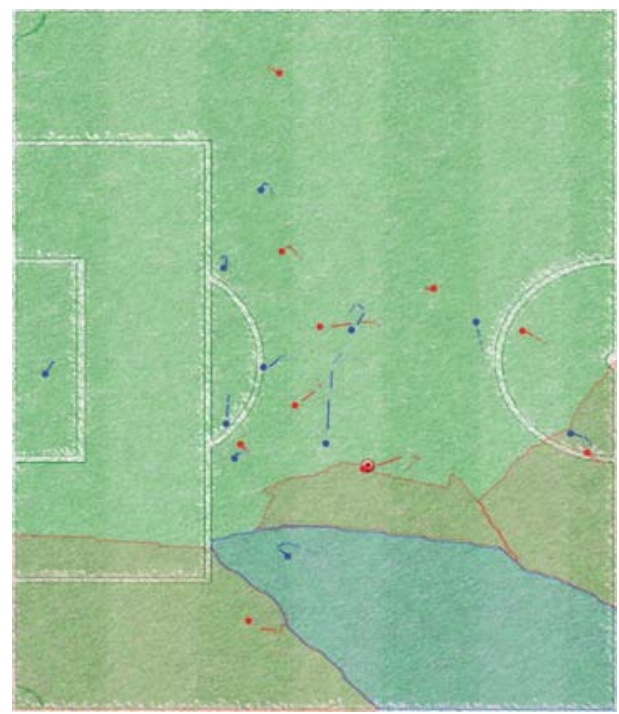

(a) Observed Movement

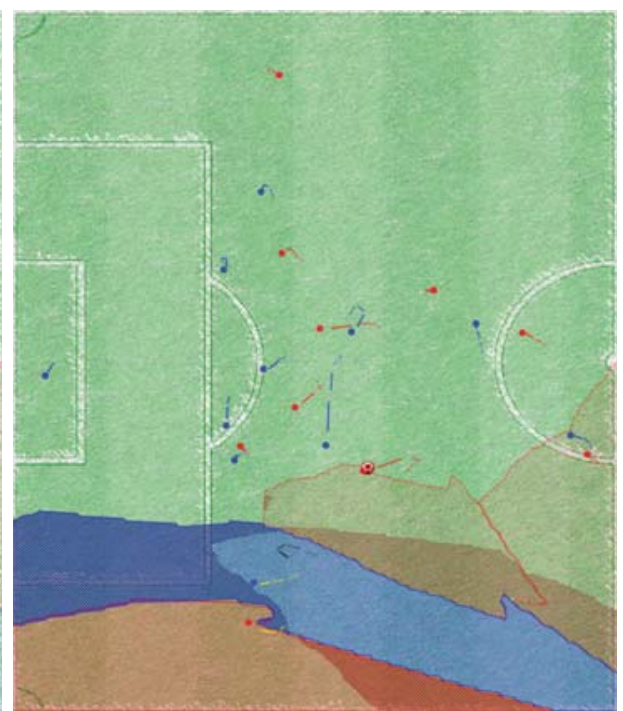

(b) Suggested player movement

Figure 6. Showing an attacking situation of the red team from a professional match of an European international soccer club competition. The free spaces of both teams are coloured in the respective team colour. Our system identified faulty movement behaviour of the bottom defending player (jersey number 15) of the blue team (a), directly before the pass towards the bottom attacking player of the red team. Our system proposes an improved movement trajectory for the defending player (b) which would have allowed him to keep control of the important free space towards his own goal. Each hatched areas indicates free spaces that a player is now controlling after the improved movement.

suggested player movement in 35 out of 42 cases $(83.33 \%)$. Interestingly, the experts did not always agree among each other when manually classifying the suggested player movement (5 out of 14 situations). We believe this level of disagreement is to be expected given individual experience and coaching strategies of involved experts. Overall, we rate it as a promising result. Table 1 shows the experts' assessment for each of the single situation. The experts highly appreciated that the system detects possible faulty movement behaviour and suggests improved player movement. The focus of our work on defensive behaviour was seen appropriate as the analysis of defensive behaviour takes an important role during match preparation. Overall, each evaluation including interviews and discussion lasted around 2 to 3 hours. According to our experts, the suggestions are very useful as starting point while they approved the possibility to integrate their expert knowledge interactively in the system by moving the players around via drag-and-drop. As our proposed system detects region-based faulty movement behaviour, all experts see great potential for an improved training process, to increase a team's performance when directly working with the identified mistakes. One expert especially mentioned the intuitive setup (exact quote) of our visual storytelling as being very beneficial for coaches and analysts. The other experts agree and added that they consider the way we communicate which player could have altered his or her movement is clearly understandable. For future work, the experts mentioned the potential of extending this work to calculate optimal solutions based on the movement of all players. Currently, we mainly focus on the player losing the most control over an important free space. Furthermore, the experts see big potential in aggregating identified faulty movement behaviour over several matches, for example, to detect players that are prone to certain mistakes.

\section{Discussion and conclusion}

We presented an approach for effective what-if analyses in soccer covering three aspects:

(1) the detection of faulty movement in space and time as described in Section 2.2.1;

(2) the computation and prediction of realistic movement alternatives proposed in Section 2.2.2;

(3) the improvement of the everyday work of coaches and video analysts by realistic what-if analyses evaluated in Section 3.

We proofed the effectiveness of our detection of relevant scenes as well as suggested player repositioning. Our approach is based on a realistic movement model, and our prediction is easily explainable and hence, expected to show acceptance by analysts and players.

We performed our what-if analysis on our described dataset of 65 matches, where the initial detection of faulty movement behaviour as well as the suggestion of improved player movement averaged on 27 minutes per match. Once calculated, loading every detected scene in a match takes less than a second. Furthermore, our system is expected to significantly accelerate the analysis process by automatically identifying relevant situations instead of a tedious manual analysis of every scene.

For future work, we plan to collect and gather data of when and, if so, where analysts are interactively shifting players to other positions. Afterwards, we can use this resulting training data to make use of machine learning algorithms to further evaluate and refine our methods. Manual feedback will be supportive for the 


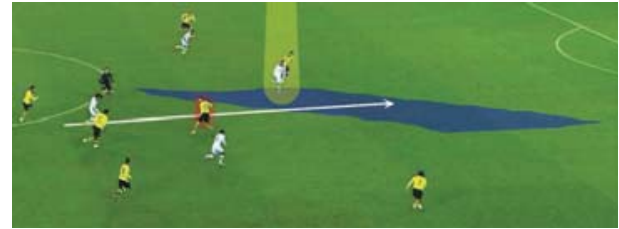

(a) Observed Movement

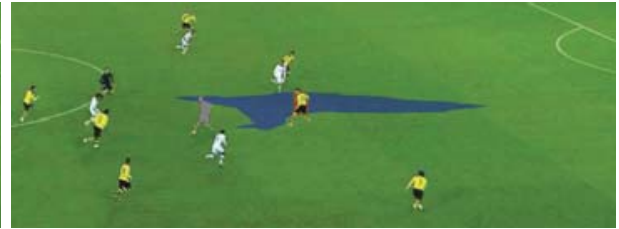

(b) Suggested player position

Figure 7. Example outcome of our proposed alteration technique used in a real video recording. In this situation, a player (highlighted in red colour) noticed his wrong location too late. If the player had positioned himself better, the free space of the opposing player would be much less severe.

analysis process as the computer does not know the individual and collective strengths and our current approach focuses on individual players instead of the complete team. Further research has to be done in the consideration of all players in the repositioning process. This non-trivial challenge with all player positions depending on all other player positions needs huge computational power and a tight interaction loop with the domain experts. The question is how far automatic processes can help here, as domain experts might not agree on the best positioning for a given situation.

So far, we restricted our studies to defensive behaviour, in order to study the elements of the algorithmic workflow in-depth. In future studies, we will work on including offensive behaviour being a straightforward extension to the approach presented in this article. We are furthermore aiming for the presentation of analysis results in the visual domain of the soccer experts. Consequently, we are working on ways to integrate our proposed approach interactively in video recordings using the approach of Bring it to the pitch (Stein et al. (2018)). The envisioned outcome is depicted in Figure 7 showing the inline video editing revealing faulty movement and proposed repositionings.

The high complexity of these what-if analyses results in an ill-defined problem with strong needs for a computer-aided visual analysis process. In more than few cases the experts do not agree among themselves identifying and resolving faulty movement. We see our work as the foundation towards a better transformation from gameplay to strategic improvements via visual predictive analytics. We envision coaches being driven by data-based decisions in their training and tactics supported by visual data analytics. The discussions on team performance will be based on data insights and not on pure intuitions.

\section{Acknowledgments}

We thank Thorsten Breitkreutz for the implementation and Johannes Häußler for the valuable feedback and discussions.

\section{Disclosure statement}

No potential conflict of interest was reported by the authors.

\section{ORCID}

Rui Marcelino (D) http://orcid.org/0000-0001-8717-3243

Michael Grossniklaus (iD) http://orcid.org/0000-0003-1609-2221

\section{References}

Abade, E. A., Gonçalves, B. V., Leite, N. M., \& Sampaio, J. E. (2014). Timemotion and physiological profile of football training sessions performed by under-15, under-17, and under-19 elite portuguese players. International Journal of Sports Physiology and Performance, 9(3), 463-470.

Alexander, J. P., Spencer, B., Mara, J. K., \& Robertson, S. (2019). Collective team behaviour of australian rules football during phases of match play. Journal of Sports Sciences, 37(3), 237-243.

BBC. (2018, June 27). Fifa's hi-tech world cup 'performance tracker'. Retrieved from https://www.bbc.com/news/av/technology-44546889/ fifa-s-hi-tech-world-cup-performance-tracker

Benito Santos, A., Theron, R., Losada, A., Sampaio, J. E., \& Lago-Peñas, C. (2018). Data-driven visual performance analysis in soccer: An exploratory prototype. Frontiers in Psychology, 9, 2416. Retrieved from https:// www.frontiersin.org/article/10.3389/fpsyg.2018.02416

Boren, T., \& Ramey, J. (2000). Thinking aloud: Reconciling theory and practice. IEEE Transactions on Professional Communication, 43(3), 261-278.

Castellano, J., Puente, A., Echeazarra, I., Usabiaga, O., \& Casamichana, D. (2016, January). Number of players and relative pitch area per player: Comparing their influence on heart rate and physical demands in under-12 and under-13 football players. PloS One, 11(1), 1-13.

Clemente, M. F., Couceiro, S. M., Martins, F. M., Mendes, R., \& Figueiredo, A. J. (2013). Measuring collective behaviour in football teams: Inspecting the impact of each half of the match on ball possession. International Journal of Performance Analysis in Sport, 13(3), 678-689.

Dobson, S., \& Goddard, J. A. (2001). The economics of football. Cambridge: Cambridge University Press.

Ericsson, K. A., \& Simon, H. A. (1984). Protocol analysis. Cambridge, MA: MIT Press.

FIFA. (2018, June 27). Facts and figures: Impact and legacy of 2018 fifa world cup russia. Retrieved from https://resources.fifa.com $/ \mathrm{mm} /$ document/tournament/competition/02/86/77/63/ Impactandlegacyof2018FIFAWorldCupENneutral.pdf

Folgado, H., Bravo, J., Pereira, P., \& Sampaio, J. (2019). Towards the use of multidimensional performance indicators in football small-sided games: the effects of pitch orientation. Journal of Sports Sciences, 37(9), 1064-1071. (PMID: 30426856).

Frencken, W. G., Lemmink, K. A., \& Delleman, N. J. (2010). Soccer-specific accuracy and validity of the local position measurement (lpm) system. Journal of Science and Medicine in Sport, 13(6), 641-645.

Gonçalves, B., Marcelino, R., Torres-Ronda, L., Torrents, C., \& Sampaio, J. (2016). Effects of emphasising opposition and cooperation on collective movement behaviour during football small-sided games. Journal of Sports Sciences, 34(14), 1346-1354(PMID: 26928336).

Guidotti, R., Monreale, A., Ruggieri, S., Turini, F., Giannotti, F., \& Pedreschi, D. (2019). A survey of methods for explaining black box models. ACM Computing Surveys, 51(5), 93:1-93: 42.

Johnston, R. J., Watsford, M. L., Kelly, S. J., Pine, M. J., \& Spurrs, R. W. (2014). Validity and interunit reliability of $10 \mathrm{hz}$ and $15 \mathrm{hz}$ gps units for assessing athlete movement demands. The Journal of Strength \& Conditioning Research, 28(6), 1649-1655.

Krustrup, P., Mohr, M., Ellingsgaard, H., \& Bangsbo, J. (2005). Physical demands during an elite female soccer game: importance of training status. Medicine and Science in Sports and Exercise, 37(7), 1242-1248. 
Le, H. M., Carr, P., Yue, Y., \& Lucey, P. (2017). Data-driven ghosting using deep imitation learning. Proceeding of the 11th MIT Sloan Sports Analytics Conference, Boston, MA. Boston: MIT.

Link, D. (2018). Data analytics in professional soccer - performance analysis based on spatiotemporal tracking data. Berlin: Springer Vieweg doi:10.1007/978-3-658-21177-6

Redwood-Brown, A., Cranton, W., \& Sunderland, C. (2012). Validation of a real-time video analysis system for soccer. International Journal of Sports Medicine, 33(08), 635-640.

Rein, R., \& Memmert, D. (2016, August 24). Big data and tactical analysis in elite soccer: future challenges and opportunities for sports science. SpringerPlus, 5(1), 1410.

Rein, R., Raabe, D., \& Memmert, D. (2017). Which pass is better?" nove approaches to assess passing effectiveness in elite soccer. Human Movement Science, 55, 172-181. Retrieved from http://www.sciencedir ect.com/science/article/pii/S0167945716302676

Sathyan, T., Shuttleworth, R., Hedley, M., \& Davids, K. (2012). Validity and reliability of a radio positioning system for tracking athletes in indoor and outdoor team sports. Behavior Research Methods, 44(4), 1108-1114.

Seidl, T., Cherukumudi, A., Hartnett, A., Carr, P., \& Lucey, P. (2018). Bhostgusters: Realtime interactive play sketching with synthesized nba defenses. Proceeding of the 12th MIT Sloan Sports Analytics Conference, Boston, MA. Boston: MIT.

Sha, L., Lucey, P., Yue, Y., Wei, X., Hobbs, J., Rohlf, C., \& Sridharan, S. (2018). Interactive sports analytics: An intelligent interface for utilizing trajectories for interactive sports play retrieval and analytics. ACM Transactions on Computer-human Interaction : A Publication of the Association for Computing Machinery, 25(2), 13:1-13: 32.

Stein, M., Janetzko, H., Breitkreutz, T., Seebacher, D., Schreck, T. Grossniklaus, M., ... Keim, D. A. (2016). Director's cut: Analysis and annotation of soccer matches. IEEE Computer Graphics and Applications, 36(5), 50-60.

Stein, M., Janetzko, H., Lamprecht, A., Breitkreutz, T., Zimmermann, P., Goldlücke, B., ... Keim, D. A. (2018). Bring it to the pitch: Combining video and movement data to enhance team sport analysis. IEEE Transactions on Visualization and Computer Graphics, 24(1), 13-22.

Stein, M., Janetzko, H., Seebacher, D., Jäger, A., Nagel, M., Hölsch, J., ... Grossniklaus, M. (2017). How to make sense of team sport data: From acquisition to data modeling and research aspects. Data, 2(1), 2.

Valter, D. S., Adam, C., Barry, M., \& Marco, C. (2006). Validation of prozone ${ }^{\circledR}$ : A new video-based performance analysis system. International Journal of Performance Analysis in Sport, 6(1), 108-119.

Varley, M. C., Fairweather, I. H., \& Aughey, R. J. (2012). Validity and reliability of gps for measuring instantaneous velocity during acceleration, deceleration, and constant motion. Journal of Sports Sciences, 30 (2), 121-127.

Waldron, M., Worsfold, P., Twist, C., \& Lamb, K. (2011). Concurrent validity and test-retest reliability of a global positioning system (GPS) and timing gates to assess sprint performance variables. Journal of Sports Sciences, 29(15), 1613-1619. 